\title{
Efficient Range Query Retrieval for Non-Uniform Data Distributions
}

\author{
Salahadin Mohammed \\ Department of Computer Technology \\ Monash University, Caulfield, Australia \\ salah@ct.monash.edu.au
}

\author{
Evan P. Harris \\ Kotagiri Ramamohanarao \\ Dept. of Computer Science \& Software Engineering \\ The University of Melbourne, Victoria 3010, Australia \\ evan@cs.mu.oz.au \\ rao@cs.mu.oz.au
}

\begin{abstract}
Answering range queries is a common database operation. Methods based on hashing techniques to minimise the cost of answering range queries by taking the query distribution into account have previously been proposed. These methods have all assumed a uniform distribution of data to disk pages to achieve good performance. This assumption makes them less useful in practice because most real data distributions are non-uniform. In this paper, we discuss a method to eliminate this restriction. Extensive experimentation using a multi-dimensional file structure, the BANG file, indicates that our method results in good performance for all data distributions. In one case an improvement of over 36 times was achieved without compromising the storage utilisation. Our method also results in a stable and efficient file organisation. If the query distribution does not change substantially, an optimised file organisation rarely requires reorganisation.
\end{abstract}

\section{Introduction}

Partial-match range queries are concerned with the retrieval of records when a limited amount of information is provided to identify those records. Answering a partialmatch range query involves reading all the pages of the relation that may contain matching records. The number of pages retrieved depends on the algorithm used to place the records within the file. The average number of pages accessed per query can be minimised if an efficient record placement algorithm which takes the query distribution into account is used.

The placement of a record within a file is determined by zero or more of its attribute values. Attributes used in the placement of records are called organising attributes. A file whose records are placed using more than one organising attribute has a multi-dimensional file organisation.

The cost of a query is often measured in terms of the number of disk pages accessed to answer the query. The aim of this paper is to describe a method of clustering records such that the average response time, over all expected range queries, is minimised.

Several methods have been proposed to optimally cluster records for range queries $[1,4,6]$. The first two $[1,4]$ only considered uniform data distributions. The basic approach of the third [6] also used a uniform data distribution, but supported non-uniform data distributions by normalising the query regions based on the actual record density in a query region. That is, knowledge of both the query and data distribution is used to construct the file organisation.

In this paper, we handle the problem of non-uniform data distributions without requiring knowledge of the data distribution by using multi-dimensional file organisations which distribute records evenly even if the data distribution is nonuniform. Although we tested our approach using the BANG file $[2,3]$, it can be used with a number of other multidimensional file structures such as the nested interpolation based grid file [13], the multi-level grid file [14] and other similar file structures $[8,12]$.

In the next section we describe the BANG file. Section 3 discusses range queries, the heuristic optimisation algorith$\mathrm{m}$, minimal marginal increase [5,7], and the cost functions that we use to minimise the average range query cost. The results of our experiments are presented in Section 4 and Section 5 is the conclusion.

\section{The BANG file}

To support the efficient processing of queries for nonuniform data distributions we require a multi-dimensional file structure whose performance does not degrade in the presence of non-uniform data distributions. While the method we will describe works with a large class of such structures, in this paper we show how we can apply our method to one of these structures, the BANG file.

The BANG (Balanced And Nested Grid) file is a multidimensional file organisation, with the structure of a balanced tree. Each leaf node corresponds to a disk page containing data records and each internal node (parent) corre- 
sponds to a disk page containing information about nodes (children) one level lower in the tree.

Initially the BANG file has one leaf node and one internal node (the root). As the number of records stored in the BANG file increases more nodes are created by dividing existing nodes. When the number of records to be stored in a disk page is greater than the capacity of the disk page, its corresponding node is divided into two new nodes.

Consider a relation with $n$ attributes, $A_{0}, A_{1}, \ldots, A_{n-1}$, as an $n$-dimensional data space where each dimension corresponds to the domain of an attribute in the relation. The domain space of the relation is the Cartesian product $D_{0} \times D_{1} \times \ldots \times D_{n-1}$, where $D_{i}$ is the domain of $A_{i}$. A record is represented as a point and a node as an $n$ dimensional partition within the domain space. A node or partition is a hyper-rectangle covering $\left(d_{0}, d_{1}, \ldots, d_{n-1}\right)$, where $d_{i}$ is a closed bounded interval describing the extent of the partition in $D_{i}$. We call partitions which correspond to leaf nodes data partitions and the remainder directory partitions.

\subsection{Dividing a leaf node}

The division into two of a data partition is a recursive process. First the partition is divided into two equal sized partitions. If the contents of the two new partitions is balanced in terms of number of records, the division process ends. Otherwise, the partition with the higher number of records is successively divided until a balance is achieved.

If the division results in two equal sized partitions it is called a peer split and the two resulting partitions are called peers. If the division results in two partitions of different sizes it is called an enclosure split because one of the partitions encloses the other.

In a BANG file, as in many other multi-dimensional file structures [12-14], a peer split is achieved using binary division of the domain of one attribute. In binary division, the value which bisects the $d_{i}$ is chosen to be the split value. Its corresponding attribute is the split attribute. Records with a split attribute value less than the split value are stored in one new partition (the lower peer) and the remainder are stored in the other new partition (the upper peer).

\subsection{Partition identifier}

A partition identifier uniquely identifies a partition in the domain space. It consists of two numbers, a (binary) partition number and a partition level. We denote it as partition number:partition level.

The partition level determines the size of a partition relative to the size of the domain space. As we explained in Section 2.1, a new partition is created by successively dividing an existing partition into two. Therefore, the size of a partition is $2^{-\mu}$ of that of the domain space, where $\mu$ is a whole number. The partition level of a partition is the value of $\mu$.

The partition number defines the address of a partition in the domain space. Partition numbering can be explained using a binary tree. Each node of the binary tree consists of a partition number, a left child and a right child. The left child corresponds to the lower peer and the right child to the upper peer. Each level of a tree corresponds to a peer split. For example, after the split of an arbitrary partition, $\mathbf{p}$, the partition number of its lower peer can be defined by prepending a $\mathbf{0}$ to the partition number of $\mathbf{p}$. Similarly the partition number of its upper peer can be defined by prepending a $\mathbf{1}$ to the partition number of $\mathbf{p}$.

An enclosure split can be seen as successive peer splits performed until a balance of records to disk pages is achieved. The partition number of the enclosing partition is the same as the original partition. The partition number of the enclosed partition depends on the number of splits performed to achieve the balance and whether lower or upper peers were chosen in each split. For example, consider the splitting of a partition with (binary) partition number $\mathbf{x}$. If a balance is achieved after four successive splits in which the upper peer, upper peer, lower peer and upper peer were chosen in the respective peer splits, the partition number of the enclosed partition will be $\mathbf{1 0 1 1 x}$.

\subsection{BANG file directory}

Each internal node (directory partition) of a BANG file contains entries which correspond to nodes in the next level of the tree. Each entry consists of a child identifier and its corresponding disk page address.

Like a data partition, a directory partition is uniquely identified by a partition number and a partition level and is denoted as partition $a: b$ where $a$ is the partition number and $b$ is the partition level. A partition which corresponds to a parent node encloses all the partitions of its children.

Definition 1 Partition $u$ : $v$ encloses partition $x: y$ if $v \leq y$ and the least significant (rightmost) $v$ bits of $x$ are identical to $u$.

Definition 2 Partition $u: v$ directly encloses partition $x$ : $y$ if there is no other partition $w: z$ which encloses $x: y$ and is enclosed by $u: v$.

\subsection{Splitting a directory partition}

When the number of entries that has to be stored in a directory partition is greater than its capacity, the directory partition is divided into two and the entry for the old node in the parent node is replaced by entries for each of the $\mathrm{t}$ wo new nodes. This process is repeated if the parent node 


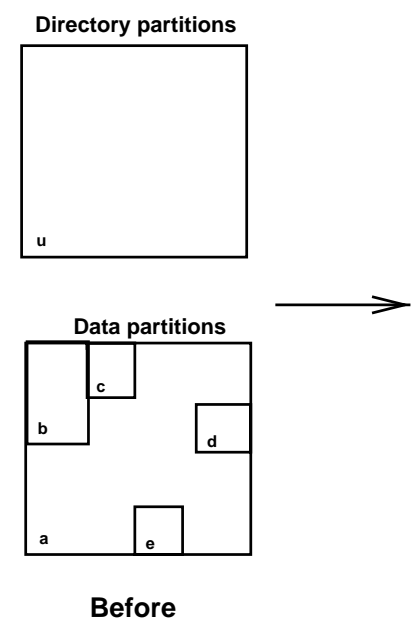

(a)
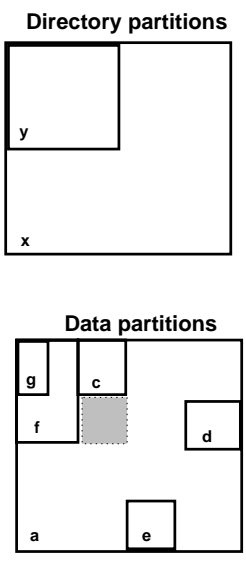

After

(b)

Figure 1. Splitting a directory.

overflows. If the partition that is divided is the root, a new root is created and entries for each of the two new nodes are placed into it.

The algorithm that is used to split a directory partition is different from that used to split data partitions. A data partition encloses data records. Data records are points, so that when a data partition is split, each of its data records will completely lie on one side or the other of the division boundary. A directory partition is a partition which encloses other partitions. Partitions are not points, so when a directory partition is split, it is possible that one or more of its component partitions lies on both sides of the division boundary.

For example, consider the BANG file given in Figure 1(a), where $\mathbf{a}, \mathbf{b}, \mathbf{c}, \mathbf{d}$ and $\mathbf{e}$ are data partitions and $\mathbf{u}$ is the root partition. Assume that the maximum number of entries that can be stored in a directory partition is five.

If, after few insertions, partition $\mathbf{b}$ is split into $\mathbf{f}$ and $\mathbf{g}$, as in Figure 1(b), the root partition $\mathbf{u}$ will overflow. If we divide $\mathbf{u}$ using the division algorithm of data partitions, it could be divided into $\mathbf{x}$ and $\mathbf{y}$. $\mathbf{x}$ encloses $\mathbf{e}$ and $\mathbf{d}$, while $\mathbf{y}$ encloses $\mathbf{f}, \mathbf{g}$ and $\mathbf{c}$. Partition $\mathbf{a}$ will lie on both sides of the division boundary. The unshaded part of $\mathbf{a}$ will lie in $\mathbf{x}$ and the shaded part will lie in $\mathbf{y}$.

To solve this problem, one option is to divide the partitions which lie on both sides of the division boundary along the division boundary. Unfortunately, this process can recursively propagate to lower level nodes. This results in a lower load factor and higher insertion cost.

Another option is to choose a division boundary such that no partition lies on both sides of the boundary. One such splitting algorithm was presented in [3]. That algorith$\mathrm{m}$ works as follows.

Assume $\mathbf{p}$ is the partition to be split. An initial boundary

which best splits the contents of $\mathbf{p}$ is chosen. Let us call this boundary $\mathbf{A}$. If there is no partition in $\mathbf{p}$ which encloses $\mathbf{A}$ or there is one partition whose boundary coincides with that of $\mathbf{A}$, then $\mathbf{p}$ is split along $\mathbf{A}$. Otherwise, search for two partitions in $\mathbf{p}$ : one which directly encloses $\mathbf{A}$, and one which does not enclose $\mathbf{A}$ but which encloses the greatest number of partitions in $\mathbf{p}$. Let us call the boundary of first partition $\mathbf{B}$ and the second one $\mathbf{C}$. Then $\mathbf{p}$ is split along $\mathbf{B}$ or $\mathbf{C}$ depending on which one of them best splits $\mathbf{p}$, that is, provides the best balance of partitions within the resulting directories.

For example, in splitting $\mathbf{u}$ in Figure 1, the initial boundary, $\mathbf{A}$, which best splits $\mathbf{u}$ is $\mathbf{y}$. We can not split $\mathbf{u}$ using this boundary because it is enclosed by $\mathbf{a}$ and does not coincide with an existing boundary. Hence, we search for boundaries $\mathbf{B}$, which directly encloses $\mathbf{A}$, and $\mathbf{C}$, which does not enclose A but encloses the greatest number of other partitions. $\mathbf{B}$ is $\mathbf{a}$ and $\mathbf{C}$ is $\mathbf{f}$. If we now split $\mathbf{u}$ along boundary $\mathbf{B}$, all its partitions will end up in one partition. If we choose $\mathbf{C}$ to split $\mathbf{u}$ the partitions $\mathbf{g}$ and $\mathbf{f}$ will be in one partition and partitions $\mathbf{a}, \mathbf{c}, \mathbf{d}$ and $\mathbf{e}$ will be in the other. Since $\mathbf{C}$ provides a better balance we split $\mathbf{u}$ along the boundary of $\mathbf{C}$.

We chose this latter algorithm because it results in a higher load factor and lower insertion cost than the former algorithm.

\subsection{Searching}

The search for a record starts at the root directory node. From the root entries, a partition which encloses the record is chosen. If there are two or more partitions which enclose the record the minimal (most specific) one is chosen. This is the one with the greater partition level.

The search then descends to the next lower level of the BANG file directory tree, following the page identifier from the chosen entry. The search procedure is repeated within the current level.

This search procedure is applied at each level of the tree until the data partition which directly encloses the record is found.

\subsection{Choice vector}

Prior to splitting a partition, a split attribute has to be chosen from the $n$ candidates. As will be demonstrated in the following sections, the order in which a domain space is partitioned significantly affects the average cost of a query. That is, the correct ordering of split attributes minimises the average cost of a query.

A description of the order of the split attributes used in partitioning a domain space is specified in a vector known as a choice vector. Each element of a choice vector is a split attribute. For example, in a BANG file with three at- 
tributes, $A_{1} A_{0} A_{2} A_{0} A_{1} A_{0} A_{2} A_{0} A_{2} A_{0} A_{1} A_{2}$ is a possible choice vector. Let $v_{i}$ denote the $i$ th split attribute. For example, in the choice vector above $v_{0}$ is $A_{1}, v_{1}$ is $A_{0}$, and so on. Any partitions with a partition level of $k$ is peer split using $v_{k}$. If $v_{k}$ does not result in two balanced partitions, $v_{k+1}$ is also used. If $v_{k+1}$ does not result in two balanced partitions, $v_{k+2}$ is also used, and so on until a balance is achieved. This implies that the length of a choice vector must at least be equal to the highest partition level in the domain space.

Existing BANG files implicitly use choice vectors which contain equal number of entries from each attribute allocated to the choice vector in a cyclic manner. Such choice vectors are called cyclic choice vectors.

\subsection{Load factor}

To determine whether the splitting policy used to form choice vectors has any impact on the load factor, we performed a series of experiments. BANG files were created using different page sizes, record sizes, numbers of attributes, data distributions, and choice vectors. Three sets of choice vectors were chosen: one set was chosen at random, one set was composed of cyclic choice vectors, and the final set were obtained using an optimisation algorithm, minimal marginal increase (MMI) which attempts to minimise the average query cost. MMI is discussed in Section 3.3.

The data distributions used were chosen from the set: uniform, clustered, sinusoidal, and linear. More details of these distributions can be found in [10].

Our experimental results show that none of the aforementioned parameters have a noticeable effect on the load factor of the BANG file. Therefore, for a given query distribution we can use the choice vector that obtains the best performance in terms of the average query cost without increasing the storage cost.

\section{Range Queries}

Range queries are a common database operation. In a partial-match range query, a range of values is specified for one or more attributes of a relation. For example, assume that $R$ is stored in the BANG file shown Figure 2(a) and query $q$, on $R$, is

\section{select $A_{1}$ from $R$ where $A_{0}$ between 35 and 48}

A range query defines a subspace within the domain space of a relation. We call such a subspace a query space. For example, the shaded area in Figure 2(a) is the query space of $q$.

The records which answer a range query can be found in the partitions (disk pages) that overlap with the query space. We call a record which satisfies a query a $\Phi$-record of the

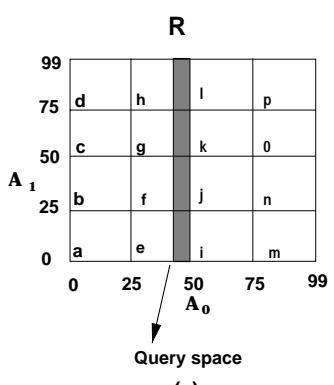

(a)

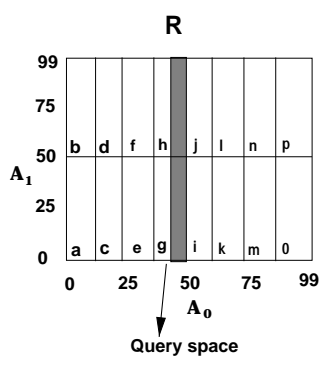

(b)

\section{Figure 2. Query space intersecting four parti- tions.}

query, and its corresponding partition a $\Phi$-partition. For example, the $\Phi$-partitions of $q$ in Figure 2(a) are $\mathbf{e}, \mathbf{f}, \mathbf{g}$ and h.

If a $\Phi$-partition is not totally enclosed by the query space, some of its records may lie outside the query space and so are not $\Phi$-records. For example, a record with a value of 30 for $A_{0}$ and a value of 40 for $A_{1}$ is not a $\Phi$-record even though it is found in $\mathbf{f}$, which is a $\Phi$-partition of $q$.

If there are two or more partitions which totally enclose a query space, only the partition which directly encloses the query space is the $\Phi$-partition. The others will not contain $\Phi$-records, so there is no need to retrieve them.

\subsection{Minimising range query costs}

The cost of a query depends on the number of disk accesses performed to answer the query. The cost of a query can be reduced if its $\Phi$-records are clustered in fewer partitions (disk pages).

The search for $\Phi$-records of a query is performed using the values of the attributes specified in the WHERE clause of the query. For example in searching for the $\Phi$-records of $q$, we use the values of $A_{0}$, because $A_{0}$ is the specified attribute in the WHERE clause of $q$. We call the attributes that we use to search for the $\Phi$-records the significant attributes.

A domain space which is partitioned to a greater degree on the most significant attributes will potentially cost less than one partitioned more on the less significant attributes or one which treats all attributes as equally significant. For example, $q$ has four $\Phi$-partitions if used with a BANG file partitioned as in Figure 2(a), but only two $\Phi$-partitions if partitioned as in Figure 2(b). This is because the domain space in Figure 2(b) is partitioned to a greater degree on $A_{0}$, which is the most significant attribute, compared with Figure 2(a) in which the domain space is partitioned using a cyclic choice vector.

Figure 2 shows how the partitioning order affects the cost of one query in a two dimensional domain space. The 
problem we are addressing is that given an $n$ dimensional domain space, an arbitrary number of queries and their probabilities, can we construct the choice vector which results in the minimal average query cost. For an arbitrary set of queries, the simpler problem of partial-match (point) retrieval is NP-hard [11]. As a result, we use an heuristic technique, minimal marginal increase (MMI), together with the cost functions presented in Section 3.2 to minimise the average query cost. MMI, which is described in Section 3.3, is a greedy algorithm and choice vectors obtained using MMI are not guaranteed to be optimal, although they have often been optimal or near-optimal for a other problems in the past [5]. In this paper we refer to choice vectors obtained using MMI as optimised choice vectors.

\subsection{Cost functions}

We define the cost of a query to be the number of disk pages accessed to answer the query. For the set of all queries $Q$, the average cost of a query is:

$$
C_{\text {avg }}=\sum_{q \in Q} p_{q} C(q)
$$

where $p_{q}$ is the probability of $q$ being asked, and $C(q)$ is the cost of answering the query $q$.

For range queries, the average cost of a query can be approximated as follows. Assume the number of data blocks of a BANG file is $2^{d^{0}}$, where $d^{0}$ is equal to the length of the choice vector, which is also the highest partition level in the domain space. Assume also that attribute $A_{i}$ appears $d_{i}^{0}$ times in the choice vector. Note that $\sum_{i=0}^{n-1} d_{i}^{0}=d^{0}$.

Let $r_{i}(q)$ be the proportion of the total range of $A_{i}$ that query $q$ specifies. For example, if the domain of $A_{i}$ is $[1,100]$, and a query, $q$, specifies the range $[2,7]$ then $r_{i}(q)=(7-2+1) / 100=0.06$. We assume that the average number of data pages accessed in answering $q$ can be approximated by

$$
C^{0}(q)=\prod_{i=0}^{n-1}\left\lceil r_{i}(q) 2^{d_{i}^{0}}\right\rceil .
$$

Let the number of disk pages which belong to directory level $h$ be $2^{d^{h}}$, where $d^{h} \leq d^{0}$ and is the length of the choice vector considered at this level. Out of these $d^{h}$ bits of the choice vector, let $d_{i}^{h}$ belong to attribute $A_{i}$. Then the average number of directory level $h$ pages accessed in answering $q$ can be approximated by

$$
C^{h}(q)=\prod_{i=0}^{n-1}\left\lceil r_{i}(q) 2^{d_{i}^{h}}\right\rceil .
$$

Combining Equations 1, 2 and 3, if there are $\Gamma$ directory levels in addition to the data pages, the average cost of the set of all queries $Q$ can be approximated by

$$
C_{\text {avg }}=\sum_{q \in Q} p_{q} \sum_{h=0}^{\Gamma} \prod_{i=0}^{n-1}\left\lceil r_{i}(q) 2^{d_{i}^{h}}\right\rceil .
$$

Previously, there was no good model available to estimate a query cost in a BANG file. Experimental studies $[2,9]$ show that the BANG file evenly distributes data records among disk blocks even when the data distribution is highly non-uniform, which makes Equation 4 a reasonable approximation of the cost.

\subsection{Minimal marginal increase}

The heuristic algorithm that we use to construct good choice vectors is minimal marginal increase (MMI) [5,7]. It works as follows.

Start with a choice vector of length zero. Tentatively allocate the first entry of the choice vector to $A_{0}$ and compute the average query cost using Equation 4 . Then compute the average query cost giving the same choice vector entry to $A_{1}$ instead, and then to $A_{2}$, and so on, until all the attributes have been tried. Choose the attribute which gives the lowest average query cost and permanently allocate the first entry of the choice vector to it. Then repeat this process for the second entry of the choice vector, then for the third, and so on, until all the entries of the choice vector are allocated. This choice vector is then used to partition the domain space.

Although the cost functions take all levels of the BANG file into account, this algorithm works primarily to optimise at the data level. This results in the directory levels also being optimised because the directory levels exist to support the data level, and their choice vectors are prefixes of the choice vector of the data level.

We use MMI because it is fast, it maintains the dynamic properties of the BANG file by extending the choice vector one bit at a time, and it has performed well in the past on similar problems $[4,5,7,9]$.

\section{Experimental Results}

In this section we present the results of experiments comparing the performance of the optimised and cyclic choice vectors.

The first set of results shows the performance of the optimised and the cyclic choice vectors on different data and query distributions. The second set of results shows the effect of the number of attributes on the performance of both choice vectors. The third and fourth sets show the effect of the file size and page size on the performance of the cyclic and optimised choice vectors. The fifth set of results show the effect of the page size on CPU time. 
Query distributions change over time. A choice vector optimised for one query distribution may not perform as well if the query distribution changes. The sixth set of results demonstrate the stability of the optimised choice vector. It answers the question of how frequently we need to compute new optimised choice vectors. The final set of results show how the performance of the optimised choice vector is affected as the average query size changes.

\subsection{Environment}

We implemented a BANG file with our extension of using a choice vector during partition splitting. Our implementation also includes the minimality and extensibility of pages described in [3].

In each experiment we used 16 randomly generated queries and assigned each of them a randomly generated probability. Unless specified, we used a page size of 1024 bytes, four integer attributes per record and one million randomly generated records per relation (BANG file). We ran all our experiments on a SPARCstation 20 running SunOS 5.5.1.

The data distributions used were uniform, clustered regions, a linear correlation, and a non-linear correlation function (a sine wave). More details of these distributions can be found in [10]. We refer to them as uniform, clustered, linear and sinusoidal, respectively.

In the experiments in which a relation of four attributes was involved, up to four different query distributions were used. The domain of each attribute is between 0 and $1048575\left(2^{20}-1\right)$. These query distributions were generated randomly and are referred to as $Q D_{1}, Q D_{2}, Q D_{3}$ and $Q D_{4}$. They are presented in detail in [10].

\subsection{Optimised choice vector}

Representative examples of the effect of using the optimised and cyclic choice vectors on the average query cost using different data and query distributions are shown in Tables 1 and 2. More results are presented in [10]. The first column in each of these tables shows the query distribution used. The second and the fourth columns correspond to the cyclic choice vector and show the cost in disk page accesses and time taken, respectively. Similarly, the third and fifth columns show the costs corresponding to the optimised choice vector. The improvement in the number of disk page accesses and time taken when using the optimised choice vector rather than the cyclic choice vector is shown in the final two columns.

In all the experiments that we performed the optimised choice vector performed better than the cyclic choice vector. The improvement is lower for the non-uniform data distributions due to correlation of the attributes. The value

\begin{tabular}{ccccccc}
\hline Query & \multicolumn{2}{c}{ Pages accessed } & \multicolumn{2}{c}{ Time (msec) } & \multicolumn{2}{c}{ Improvement } \\
\cline { 2 - 7 } Dist. & Cylic & Opt. & Cyclic & Opt. & Pages & Time \\
\hline$Q D_{1}$ & 77.06 & 7.32 & 624.76 & 80.54 & 10.52 & 7.75 \\
$Q D_{2}$ & 166.96 & 18.96 & 1147.5 & 172.67 & 8.80 & 6.64 \\
$Q D_{3}$ & 75.72 & 14.52 & 599.87 & 147.75 & 5.22 & 4.06 \\
$Q D_{4}$ & 118.34 & 13.78 & 914.36 & 138.12 & 8.59 & 6.62 \\
\hline
\end{tabular}

Table 1. Average query cost for a uniform data distribution.

\begin{tabular}{ccccccc}
\hline Query & \multicolumn{2}{c}{ Pages accessed } & \multicolumn{2}{c}{ Time (msec) } & \multicolumn{2}{c}{ Improvement } \\
\cline { 2 - 7 } Dist. & Cylic & Opt. & Cyclic & Opt. & Pages & Time \\
\hline$Q D_{1}$ & 7.17 & 5.05 & 66.39 & 61.86 & 1.42 & 1.07 \\
$Q D_{2}$ & 21.02 & 7.47 & 168.27 & 70.08 & 2.81 & 2.40 \\
$Q D_{3}$ & 9.16 & 6.79 & 76.17 & 62.64 & 1.35 & 1.22 \\
$Q D_{4}$ & 17.25 & 9.02 & 140.39 & 87.26 & 1.91 & 1.61 \\
\hline
\end{tabular}

\section{Table 2. Average query cost for a linear data distribution.}

of the first attribute was randomly generated and then used to generate the values of the remainder of the attributes.

\subsection{Number of attributes}

As the number of attributes increases, the number of attributes that are specified in few or no queries (insignificant attributes) is likely to increase. Therefore, the degree of the domain space partitioned based on these attributes also increases if the cyclic choice vector is used. As a result, the performance improvement achieved by using the optimised choice vector instead of the cyclic choice vector should also increase.

Table 3 shows experimental results obtained using BANG files with different numbers of attributes for different query distributions. The first column of the table shows the number of attributes in each BANG file. The second column shows the average number of disk page accesses required when the cyclic choice vector was used. The third column shows the average number of disk page accesses required when the optimised choice vector was used. The last column shows the improvement achieved using the optimised choice vector rather than the cyclic choice vector. The table shows that as the number of attributes increases the improvement increases, as we expect.

The ratio of the significant attributes to the insignificant attributes is an important factor in the improvement that is achieved. As this ratio increases, the performance improvement of the optimised choice vector over the cyclic choice vector decreases. That is, the total number of attributes in a relation is not as important as the proportion of significant 


\begin{tabular}{cccc}
\hline Number of & \multicolumn{2}{c}{ Pages } & \\
\cline { 2 - 3 } Attributes & Cyclic & Opt. & Gain \\
\hline 2 & 107.14 & 22.58 & 4.74 \\
2 & 101.15 & 18.80 & 5.38 \\
2 & 112.51 & 17.98 & 6.25 \\
2 & 96.13 & 22.76 & 4.22 \\
3 & 260.72 & 22.89 & 11.39 \\
3 & 244.80 & 29.94 & 8.18 \\
3 & 304.52 & 23.86 & 12.76 \\
3 & 317.59 & 19.52 & 16.26 \\
4 & 77.06 & 7.30 & 10.52 \\
4 & 166.96 & 18.96 & 8.80 \\
4 & 75.72 & 14.52 & 5.22 \\
4 & 118.34 & 13.78 & 8.59 \\
8 & 255.06 & 7.05 & 36.17 \\
8 & 274.08 & 11.21 & 24.45 \\
8 & 260.67 & 15.01 & 17.37 \\
8 & 214.97 & 10.16 & 21.16 \\
\hline
\end{tabular}

Table 3. Effect of the number of attributes on the average query cost.

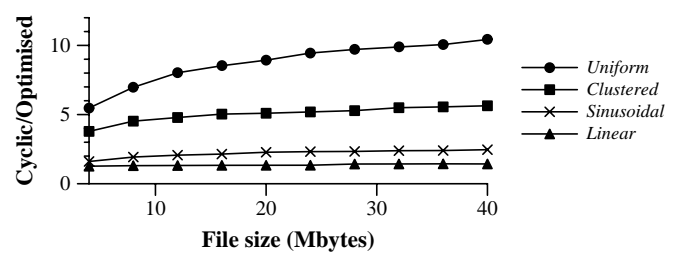

Figure 3. Effect of file size on relative performance.

attributes. In Table 3 the optimised choice vector performs better when the number of attributes is three than when it is four. This is because the proportion of significant attributes is $\frac{1}{3}$ in the former case and $\frac{1}{2}$ in the latter case. This can be determined by examining the query distributions.

\subsection{File size}

To study the effect of the file size on the performance of the optimised and cyclic choice vectors, we experimented with files ranging in size from 4 Mbytes to 40 Mbytes. The experiments were repeated using the uniform, clustered, sinusoidal and linear data distributions. The results are shown in Figure 3. The vertical axis of Figure 3 represents the average query cost ratio $\frac{\text { cyclic }}{\text { optimised }}$ and the horizontal axis represents the file size in Mbytes.

In all the experiments the optimised choice vector consistently performed better than the cyclic choice vector as can be seen in Figure 3. The improvement increased as the file size increased.

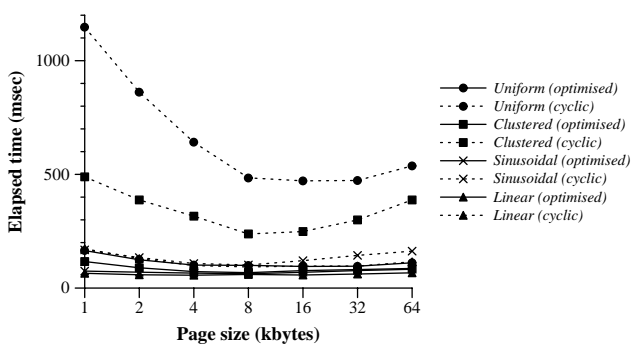

Figure 4. Effect of page size on performance.

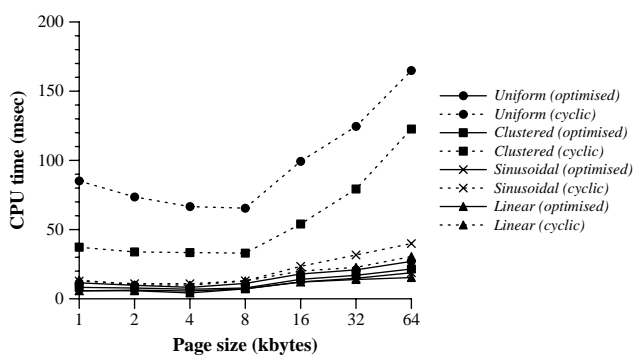

Figure 5. Effect of page size on CPU time.

\subsection{Page size and elapsed time}

We performed a series of experiments to study the effect of the page size on the performance of the optimised and cyclic choice vectors. Page sizes between 1 and $64 \mathrm{k}$ bytes were used. The experiments were repeated using the uniform, clustered, sinusoidal and linear data distributions. The results are shown in Figure 4.

As can be seen in Figure 4, the optimised choice vector performs better than the cyclic choice vector for all page sizes. The results show that the performance improvement is greater with smaller page sizes. This is because smaller pages result in higher number of pages and page splits, so the improvement gained by using a better splitting policy is greater.

In these results, the minimum elapsed time was achieved when the page size was 8 kbytes.

\subsection{Page size and CPU time}

We performed experiments to determine which page size results in the minimum CPU time, that is, the minimum time spent searching the contents of pages rather than waiting for the disk. Again, we performed our experiments using pages between 1 and 64 kbytes. The results are shown in Figure 5 .

The results indicate that for all the distributions, the minimum CPU time is generally achieved when the page size is 4 kbytes. 


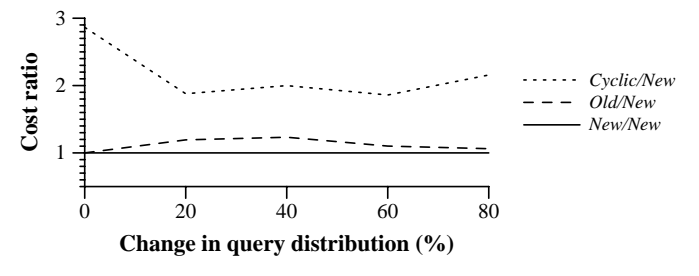

Figure 6. Stability of the optimised choice vector using $Q D_{1}$ and the sinusoidal data distribution.

\subsection{Stability}

Query distributions can change over time. A choice vector optimised for a given query distribution may perform worse than the cyclic choice vector if the query distribution changes significantly. In order to study the stability of our optimised choice vectors, we performed a series of experiments to determine the change in performance when the query distribution changes. These experiments were similar to those performed in [9].

We state that each query distribution is changed by $x \%$ if each query probability, $p$, is randomly changed to be in the range $p \times\left(1 \pm \frac{x}{100}\right)$ prior to the whole query distribution being normalised.

Figure 6 contains an example of how the average query cost is affected when the probability of each query changes by up to $80 \%$. Three average query cost ratios are shown, using dotted, dashed and solid lines. The dotted line ("Cyclic/New") corresponds to comparing the average query cost of a BANG file built using a cyclic choice vector with that of a BANG file built using a choice vector optimised for the new, changed, query distribution. The dashed line ("Old/New") correspond to a BANG file which was built using an optimised choice vector determined by using the original query distribution. The solid line ("New/New") corresponds to BANG files built using an optimised choice vector determined using the changed probability distribution. More results are presented in [10].

In some of the experiments, using a choice vector produced using the original query distribution was better than using one produced by the changed query distribution. This is because the new choice vector is not optimal. This can occur because MMI does not guarantee to produce the optimal choice vector, and Equation 4 defines a good approximation of the actual cost, not the exact cost.

Our results show that the performance of the optimised choice vector of the original query distribution is almost as good as that of the changed query distribution even when the distribution changes by $80 \%$. As a result, we conclude an optimised BANG file needs to be reorganised rarely, and only when the query distribution changes drastically.

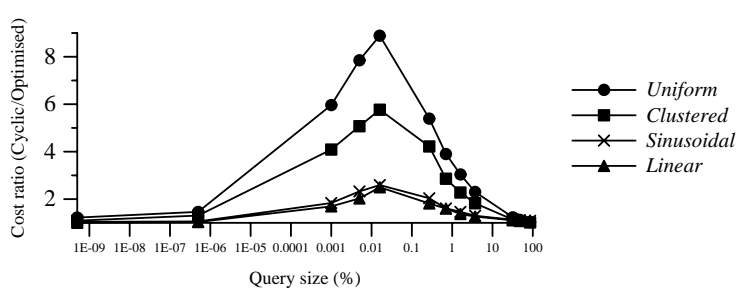

Figure 7. Effect of query area size on relative performance.

\subsection{Query space size}

When the query space is the whole domain space, we must access all the pages of the file regardless of the choice vector used. When the query is a point query the number of page accesses performed using both the optimized and cyclic choice vectors will be the same because there is precisely one destination page. Therefore, there is no advantage in using an optimised choice vector for these two extreme queries.

We performed experiments in order to study the effect of the query space size on the performance using the optimised choice vector. Figure 7 shows the performance of the optimised choice vector compared to the cyclic choice vector as the query space size changes. Each line in the figure corresponds to a different data distribution. The vertical axis of the figure represents the cost ratio $\frac{\text { cyclic }}{\text { optimised }}$ and the horizontal axis represents the percentage the query space size is to the domain space size. The results show that there is a large range of query space sizes where using an optimised choice vector produces far better results than using the cyclic choice vector.

\section{Conclusion}

Our study shows that given a probability distribution of range queries, an efficient physical database design can be created by using minimal marginal increase and Equation 4 . Unlike previous approaches, our approach is not limited to a uniform data distribution or to independently specified attributes, and the precise nature of any non-uniformity does not need to be known. We avoid these limitations by using a file structure which distributes records evenly amongst disk pages even when the data distribution is highly nonuniform. For our experiments, we used the BANG file.

When compared to the cyclic choice vector, our results show that the optimised choice vector produces more efficient physical database designs, reducing the average query cost. For example, in one of our experiments in which a BANG file of eight attributes was used, the optimised 
choice vector resulted in an improvement of a factor of 36 over the cyclic choice vector.

The improvement gained by using an optimised choice vector instead of the cyclic choice vector increases as the number of attributes increases. This is because as the number of attributes increase, the likelihood of dividing the domain space using attributes which do not occur frequently in queries is higher when the cyclic choice vector is used. This results in an inefficient physical database design. For example, in the experiments that we performed, the improvement in performance was greater when there were eight attributes in the relation than when there were two, three or four attributes. Similarly, as the ratio of attributes which occur frequently in queries to attributes which do not increases, the improvement in the performance of the optimised choice vector over the cyclic choice vector decreases.

We found that the optimised choice vector consistently performs better than the cyclic choice vector across a wide range of file and page sizes. The improvement is greater as files get larger and pages get smaller. For both types of choice vector, the CPU time taken searching pages is minimal when the page size is 4 kbytes.

The relative size of the query space also affects the performance of the optimised choice vector. For a query space size which is either equal to the whole domain space or is a point query space, both the optimised and cyclic choice vectors perform the same. However, the optimised choice vector performs better than the cyclic choice vector when the query space size is between these two extremes.

There is no need to rearrange the optimised choice vector whenever the query distribution changes by a small amount. Our experiments show that the optimised choice vector for a given query distribution will remain almost as good as the optimised choice vector built for a variation on the query distribution even when the query distribution changes by $80 \%$.

We conclude that if the query distribution is known and a file structure which evenly distributes records amongst disk pages is used regardless of the data distribution, an optimised choice vector produces an efficient physical database design. To our knowledge, this is the most practical method of storing multi-dimensional data in order to best exploit a known query distribution. We therefore recommend that such structures be incorporated into new generation database systems.

\section{Acknowledgements}

The authors were supported by Australian Research Council grants. The first author was also supported by a Monash University postgraduate scholarship. We also thank Prof. Srinivasan for his helpful comments.

\section{References}

[1] C. Y. Chen, C. C. Chang, and R. C. Lee. Optimal MMI file systems for orthogonal range queries. Information Systems, 18:37-54, 1993.

[2] M. Freeston. The BANG file: a new kind of grid file. In U. Dayal and I. Traiger, editors, Proceedings of the 1987 ACM SIGMOD International Conference on the Management of Data, pages 260-269, San Francisco, California, USA, May 1987.

[3] M. Freeston. Advances in the design of the BANG file. In Proceedings of the Third International Conference on the Foundations of Data Organization and Algorithms, Paris, France, June 1989.

[4] E. P. Harris and K. Ramamohanarao. Optimal dynamic multi-attribute hashing for range queries. BIT, 33(4):561579, 1993.

[5] E. P. Harris and K. Ramamohanarao. Generalising minimal marginal increase to cluster records in multi-dimensional data files. In J. Roddick, editor, Database Systems '99, Proceedings of the 10th Australasian Database Conference, pages 129-140, Auckland, New Zealand, Jan. 1999. Springer.

[6] J.-H. Lee, Y.-K. Lee, K.-Y. Whang, and I.-Y. Song. A region splitting strategy for physical database design of multidimensional file organizations. In Proceedings of the 23rd VLDB Conference, pages 416-425, Athens, Greece, Aug. 1997.

[7] J. W. Lloyd and K. Ramamohanarao. Partial-match retrieval for dynamic files. BIT, 22:150-168, 1982.

[8] D. B. Lomet and B. Salzberg. The hb-tree: A multi-attribute indexing method with good guaranteed performance. ACM Transactions on Database Systems, 15(4):38-71, Dec. 1990.

[9] S. Mohammed, E. P. Harris, and K. Ramamohanarao. Efficient partial-match retrieval for skewed data distributions. In J. Roddick, editor, Database Systems '99, Proceedings of the 10th Australasian Database Conference, pages 37-48, Auckland, New Zealand, Jan. 1999. Springer.

[10] S. Mohammed, E. P. Harris, and K. Ramamohanarao. Efficient range query retrieval for non-uniform data distributions. Technical Report 1999/29, Department of Computer Science and Software Engineering, The University of Melbourne, Parkville, Victoria 3052, Australia, 1999.

[11] S. Moran. On the complexity of designing optimal partialmatch retrieval systems. ACM Transactions on Database Systems, 8(4):543-551, Dec. 1983.

[12] J. Nievergelt, H. Hinterberger, and K. C. Sevcik. The grid file: An adaptive symetric multikey file structure. $A C M$ Transactions on Database Systems, 9(1):38-71, Mar. 1984.

[13] A. Ouksel and O. Mayer. A robust and efficient spatial data structure. Acta Informatica, 29, 1992.

[14] K.-Y. Whang and R. Krishnamurthy. The multilevel grid file - a dynamic hierarchical multidimensional file structure. In International Symposium on Database Systems for Advanced Applications, pages 449-459, Tokyo, Japan, Apr. 1991. 